\title{
British Association of Otolaryngologists Head and Neck Surgeons Summer Meeting, 7-8 September 2005, Edinburgh, Scotland, UK: general abstracts
}

\author{
Spontaneous resolution of initial indication for \\ tonsillectomy in adult patients, resulting in attrition from \\ tonsillectomy waiting list \\ C Y Eng, G G Browning*, J Dempster ${ }^{\dagger}$, W S McKerrow ${ }^{*}$ \\ From the Aberdeen Royal Infirmary, Aberdeen, the *Glasgow \\ Royal Infirmary, Glasgow, the ${ }^{\dagger}$ Crosshouse Hospital, Kilmarnock, \\ and the ${ }^{\star}$ Raigmore Hospital, Inverness, Scotland, UK
}

\section{Introduction}

Concern over the risk of variant Creutsfeld-Jacob disease transmission during tonsillectomy resulted in a complete embargo on the operation until a safer strategy could be found. This provided clinicians with an opportunity to observe the spontaneous resolution rate of the initial indications for tonsillectomy in patients on the waiting list. We aimed to study this spontaneous resolution rate in adult patients on the waiting list, and to assess whether factors such as hospital, gender, age and duration on waiting list affected the final decision to undergo surgery.

\section{Method}

This was a Scottish, multi-centre, prospective, crosssectional study. The three hospitals involved were the Glasgow Royal Infirmary, Glasgow, the Crosshouse Hospital, Kilmarnock, and the Raigmore Hospital, Inverness. Patients aged 15 years and over whose names were placed on the waiting list for tonsillectomy from March 1995 to December 2001 and who were still waiting for their operation were invited to attend a consultant-led reassessment clinic. This reassessment clinic ran for 15 months from March 2003 to May 2004

\section{Results}

A total of 189 patients were identified. The male to female ratio was 1:2.5. One hundred and forty-six ( 77.2 per cent) patients attended the reassessment clinic. All 43 patients who failed to attend the reassessment clinic were removed from the waiting list. A total of 72 (38.3 per cent) patients were removed from the waiting list. The difference in attrition rate between hospitals was statistically significant $(p<0.000)$. Patient gender did not affect the final decision to undergo surgery. Younger patients tended to remain on the waiting list more than older patients $(p<0.000)$. Patients who had waited for a shorter period of time also tended to remain on the waiting list $(p<0.000)$.

\section{Conclusions}

Our results showed that the majority of patients were on the waiting list for less than a year, and a significant proportion of patients did not require tonsillectomy at reassessment. It was noted that there was a statistically significant difference in the waiting list attrition rate between the three hospitals. This suggested that there were variations in practice among the hospitals and is consistent with the results of previous studies. Our study results support the usefulness of patients undergoing a period of observation before being listed for tonsillectomy; our results also support tonsillectomy being carried out as soon as possible following listing for surgery in order to maximize its benefit.

Endoscopic suction diathermy adenoidectomy: the end of a blind procedure?

M Jaramillo, D Owens*, M W Saunders ${ }^{\dagger}$

From the Southmead Hospital, Bristol, the *University Hospital of Wales, Cardiff, and ${ }^{\dagger}$ St Michaels Hospital, Bristol, UK

\section{Introduction}

We describe various techniques to improve the view of the surgical field while performing suction diathermy adenoid ablation.

\section{Method}

We present a detailed description of surgical technique, emphasizing differences in ease, view and operation time, for the conventional adenoidectomy technique used in the UK. The process of improving the operative field view while performing suction diathermy adenoidectomy is depicted. The main aspects considered are: different options for bending the suction coagulator shaft; change in the laryngeal mirror angle and size; and, ultimately, use of a $90-120^{\circ}$ endoscope and digital imaging system stack.

Results

Using audit data, we summarize the local experience at the Bristol Children's Hospital.

Conclusions

Alterations in the suction coagulator shaft and use of an endoscopy and imaging system substantially improve visualization of the operative field during suction diathermy adenoidectomy.

\section{A completed audit cycle on post tonsillectomy haemorrhage}

F Javed, M Sadri, J Uddin, S Mortimore, D A Parker

From the Department of Otorhinolaryngology, Derbyshire Royal Infirmary, Derby Hospitals, NHS Foundation Trust, Derby, UK

\section{Objective}

To compare the post-operative haemorrhage rate after coblation tonsillectomy and routine dissection tonsillectomy. The initial cycle of the audit was undertaken prior to the National Prospective Tonsillectomy Audit.

Setting

A large sized district general hospital, located in the centre of England. 


\section{Method}

In the first audit period, 441 sequential tonsillectomies in adult and paediatric age groups between January and September 2002 were retrospectively reviewed. Haemorrhage rate subsequent to coblation was compared with cold steel and diathermy dissection tonsillectomies. Coblation tonsillectomy was subsequently abandoned in our unit. In the second audit period, all tonsillectomies (416 cases) between July 2003 and August 2004 were examined retrospectively. Statistical analysis was performed using chisquared test.

Results

In the first audit period, 191/441 (43.3\%) patients underwent coblation and 250/441 (56.7\%) underwent dissection tonsillectomy. The primary haemorrhage rate in the coblation group was $2.1 \%(4 / 191)$ compared to $1.6 \%(4 / 250)$ in the dissection group $(p=0.697)$. In the second cycle with no coblation procedures, $6 / 416(1.4 \%)$ patients suffered from primary haemorrhage (compared with $8 / 441$ in the first cycle, $p=0.666$ ). Secondary haemorrhage, was seen in the first period amongst $20 / 250$ patients $(8.0 \%)$ in the dissection group and $48 / 191(25.1 \%)$ in the coblation group $(p<0.001)$. In the second cycle, after coblation was discontinued, the secondary haemorrhage rate fell significantly $(p<0.001)$ to $5.8 \%(24 / 416)$.

\section{Conclusion}

A significant reduction in post-tonsillectomy secondary haemorrhage rate was observed after coblation tonsillectomy was abandoned in our department.

\section{Patient satisfaction with the ENT out-patient clinic}

H Khan, K Mattam, N Malik, S Singh, J Matthews, F Wilson From the Department of ENT, New Cross Hospital, Wolverhampton, UK

\section{Introduction}

The aim of this study was to assess and quantify the degree of patient satisfaction with an ENT out-patient clinic and also to investigate if patient dissatisfaction had any relation to the waiting time in the clinic and to the consultation process with different grades of doctors.

\section{Method}

A prospective, cross-sectional survey was carried out of 200 patients, using a validated out-patient satisfaction questionnaire with a five-point Likert scale, with positively and negatively worded questions to avoid acquiescent bias. Overall patient satisfaction was assessed based on a 10-point visual analogue scale (VAS).

\section{Results}

Eighty-five per cent of patients were very satisfied (VAS > 5; mean score, 9.0). Five per cent of patients were not satisfied with the service (VAS $\leq 5$; mean score, 2.5 ). No difference was found in degree of patient satisfaction regarding the grade of doctor or waiting time in the clinic.

\section{Conclusions}

Our study attempted to quantify patients' satisfaction with ENT out-patient clinic practice. The results indicate that the current practice was satisfactory. The study outcome also implies that a questionnaire-based analysis can be used to objectively identify the effect of any significant change in practice in an out-patient clinic. The level of patient satisfaction with the ENT out-patient clinic was not influenced by the duration of waiting time or the seniority of the doctor. Also, no relationship was found between patients' ethnic origin and their level of satisfaction, dispelling the myth that Asian patients are more dissatisfied than white patients.

\section{Results of adult day-case tonsillectomy}

E Kiverniti, P Tostevin, M Vouros*, V Moore-Gillon

From St George's Hospital, London, and the *Broomfield Hospital, Chelmsford, UK

\section{Introduction}

We aimed to present current practice and to assess subjective patient satisfaction with day-case tonsillectomy.

\section{Method}

A prospective study of 99 adults was undertaken. Seventy patients satisfied the criteria for day surgery; the control group of 29 had an overnight stay. One month following tonsillectomy, all patients underwent a telephone interview regarding post-operative complications and overall satisfaction, measured on a numerical analogue scale.

\section{Results}

Sixty-two patients (62.62 per cent) underwent day surgery. Eight patients (8.08 per cent) planned to have day surgery but actually stayed overnight. Twenty-nine patients (29.29 per cent) had a planned overnight admission. In the overnight stay group, $3.5 \%$ of patients presented with postoperative bleeding on day 1 and had to be readmitted, and the overall grade of satisfaction was 8.34. In the day-case group, $3 \%$ of patients were readmitted for postoperative bleeding on day 1 , two patients had to be readmitted due to pain (2.85 per cent), and the overall grade of satisfaction was 7.84. Of the 70 patients in the day-case group, 40 were happy with day-case care (57.14 per cent) and 30 reported that in future they would opt for an overnight stay if given the choice (42.85 per cent). Twenty-nine patients remained in hospital overnight; 24 of these patients were satisfied with this ( 82.45 per cent), while five were not and would have returned home if allowed to $(17.24$ per cent). It is interesting to note that taste disturbance was noted in 31 cases (31.31 per cent), temporary in 17 cases (17.17 per cent) and persistent in 14 cases (14.14 per cent).

\section{Conclusions}

We support the practice of day-case tonsillectomy in adults, but we stress the importance of taking patients' wishes into consideration.

\section{Tonsillectomy for psoriasis: does it help?}

V Prasad, N Mani, A Suraliraj, K Burova, T J Hoare

From the Bedford Hospital NHS Trust, Bedford, UK

\section{Introduction}

We aimed to assess patient satisfaction before and after tonsillectomy for treatment of psoriasis.

\section{Method}

A review of 16 cases of psoriasis referred to the ENT department was undertaken using the validated self-administered psoriasis area and severity index (SAPASI) to determine the outcome of tonsillectomy for psoriasis.

\section{Results}

Thirteen patients successfully completed the questionnaire. Twelve patients were female and one was male; the average age was 25 years (range, 5-36 years). Seven patients reported improvement in their psoriasis following 
tonsillectomy, for both average and severe attacks. These patients were slightly better, with a reduction of SAPASI score from 27.7 pre-operatively (range, $0-72$ ) to 2.3 post-operatively when comparing attacks at their worst. Although one patient felt that the tonsillectomy had made no change to the severity of her symptoms, her SAPASI score was more that six-fold lower postoperatively for both average and severe psoriasis attacks (37.6 and 47.7 pre-operatively compared with 6.0 and 6.0 post-operatively, respectively). Two patients felt that they were worse off after the operation; however, their average SAPASI scores were less during severe attacks of psoriasis post-tonsillectomy.

\section{Conclusions}

More than 75 per cent of patients undergoing tonsillectomy for psoriasis reported a subsequent improvement in their symptoms for both average and severe attacks. None of the patients reported worsening of their SAPASI symptom score following tonsillectomy, and 70 per cent would recommend tonsillectomy as a treatment for psoriasis. Despite the small study group, we feel that offering tonsillectomy should be considered as a treatment option for psoriasis.

\section{Systematic reviews: a triumph of form over substance?}

\section{Thomas, J A Wilson*}

From the University Hospital Aintree, Liverpool, and the ${ }^{*}$ Freeman Hospital, Newcastle, UK

\section{Introduction}

We aimed to estimate the usefulness of systematic reviews in routine otolaryngology practice.

\section{Method}

The current listings in the ENT Cochrane library were reviewed. Computer-based searches were undertaken in Medline, Embase and Cinahl for otolaryngology-related meta-analyses, using 46 comprehensive terms. The abstracts, and full-text articles where needed, were reviewed.

Results

Of the 20 completed Cochrane reviews, 11 found too few data to draw conclusions; the remaining nine reviews identified deficiencies in available data and recommended further randomized, controlled trials (RCTs). The other published meta-analyses included 190 non-surgical topics and 33 surgical topics. Of the surgical topics, only three had meta-analysis from RCTs; the rest were cohort studies and case series.

\section{Conclusions}

Level 1a evidence was so lacking in otolaryngology that the added value of a systematic review, compared with a routine literature search, appeared questionable. Research commissioners need to support alternative methods for generating an appropriate evidence base for surgical interventions (e.g. decision analysis techniques to determine whether an RCT is warranted). 